Med Principles Pract 1989;1:174-176

\title{
Molecular Genetics in the Study of Human Disease
}

International Workshop sponsored by the Kuwait University Faculty of Medicine, held in Kuwait, April 9-13,1988

Dr. Sinan Taş, Department of Pathology, Faculty of Medicine, Kuwait University Kuwait

Conducted by:

V.N. Y. Chan, University of Hong Kong; J.O.D. McGee, University of Oxford, UK; R.K. Saiki, Cetus Corporation, Calif., USA; Local faculty: M. Olive, M. Şimşek, S. Taş Advances during the last few years have revolutionized the means for identifying and understanding the genetic components of disease. Molecular techniques for the study of DNA polymorphism have been available as research methods since the late 1970s but not for routine laboratory diagnosis until very recently. The classical approach in the study of DNA polymorphism - the autora-diographic analysis of the Southern-blotted genomic DNA hybridized with radiolabeled probes - has served us well. However, the process is long and complicated, and working with radioisotopes creates further problems (limited useful life of the probes, health hazards, procurement problems), precluding adoption by most clinical laboratories. Hybridization of Southern blots with nonradioisotopically labeled probes is a useful variation of the procedure. Probes that are labeled nonradioisotopically (e.g. with biotin) are stable for several months, and their detection usually takes only a few hours. Nevertheless, the blotting, hybridization and washing steps are still time-consuming and labor-intensive.

An ingenious enzymatic procedure has now revolutionized the field by allowing selective in vitro amplification within a few hours of specific genomic sequences several-million-fold [1]. Termed 'polymerase chain reaction' (PCR) because the DNA strands synthesized by DNA polymerase in each cycle of the reaction themselves serve as templates in the subsequent cycles to allow for a near logarithmic amplification of the target sequence, it was reported by R.K. Saiki and his colleagues in 1985 [2]. Subsequent improvements in the procedure, primarily with the use of a heat-stable DNA polymerase [1], were discussed and demonstrated at the workshop by R.K. Saiki. Because of the several-million-fold amplification of the specific genomic sequences, their analysis by allele-specific oligonucleotide probes can be carried out in a short time with high efficiency [1]. Recent automation of PCR using a thermocycler [1] is an additional attraction, and more improvements are anticipated, in particular in the analysis of amplified sequences (fig. 1) [see ref. 3, for example].

Molecular Genetics in the Study of Human Disease

175

$5 / 8 \mathrm{Sfr}$

$\mathrm{t} *$

Fig. 1. Electrophoretic analysis of an amplified genomic target containing a polymorphic restriction site. Lanes 1, 2 and 3 represent, respectively, DNA from subjects homozygously negative, heterozygous and homozygously positive for this restriction site. 
Fig. 2. Human papilloma virus infection detected by in situ hybridization. Viral genomes are easily seen in many nuclei in this cervical biopsy. This patient had cervical intraepithelial neoplasia III.

Thus, a simple, cost-effective means for routine clinical laboratory diagnosis of DNA alterations appears to be here, and there are problem-solving applications in almost every medical discipline. Unequivocal diagnoses can now be made readily in the laboratory not only for classical inherited diseases but also for genetic predisposition to environmental diseases, and before they are clinically apparent. Equally useful applications are found in the identification of the causative microorganisms in infectious diseases. The improved understanding of disease mechanisms, prevention and treatment which this technology will bring is eagerly awaited.

In situ hybridization (fig. 2) was another important technique discussed and demonstrated during the workshop. It is an attrac-

tive approach in analyzing patterns of gene expression in complex tissues, in virology and in several other situations where information on the localization of hybridizing targets is desirable. Here again, nonradioiso-topically labeled probes are found not only to provide higher spatial resolution than ra-diolabeled probes but also to provide simpler signal detection. Current noniso-topic in situ hybridization assays are more sensitive than radioactive procedures and can detect single copy genes on chromosomes and will detect as little as 5-10 copies of messenger RNA within intact cells [4-6]. Further improvements (in tissue preservation and hybridization strategies as well as in methods of signal measurement), however, are required before in situ hybridization assays can be considered truly quantitative. 176

Workshop Summary

\section{References}

Saiki RK, Gelfand DH, Stoffel S, et al: Primer-directed enzymatic amplification of DNA with a thermostable DNA polymerase. Science 1988; 239:487-491.

Saiki RK, Scharf S, Faloona F, et al: Enzymatic amplification of ß-globin genomic sequences and restriction site analysis for diagnosis of sickle cell anemia. Science 1985;230(4732): 1350-1354. Ta $3 / 8$ S: Strong association of a single nucleotide substitution in the 3'untranslated region of the apolipoprotein-CII gene with common hypertri-glyceridemia in Arabs. Clin Chem 1989;35:256-259.

Burns J, Graham AK, Franks C, et al: Detection of low copy human papilloma virus DNA and mRNA in routine paraffin sections of cervix by nonisotopic in situ hybridisation. J Clin Pathol 1987;40:858-864.

Bhatt B, Burns J, Flannery D, et al: Direct visualization of single copy genes on banded metaphase chromosomes by nonisotopic in situ hybridization. Nucleic Acids Res 1988;16:3951-3961. Chan VT-W, McGee JOD: Cellular oncogenes in neoplasia. J Clin Pathol 1987;40:1055-1062. 